\title{
银硫纳米簇合物的阴离子模板法合成、结构与 发光性能
}

骆耿耿 ${ }^{1}$, 王芝 ${ }^{2}$, 程丽萍 ${ }^{1,2}$, 赵全芹 ${ }^{2}$, 王兴坡 ${ }^{2}$, 孙頔 ${ }^{2 *}$

1. 华侨大学材料科学与工程学院, 厦门 361021

2. 山东大学化学与化工学院, 济南 250100

*通讯作者, E-mail: dsun@sdu.edu.cn

收稿日期: 2016-12-26; 接受日期: 2017-02-09; 网络版发表日期: 2017-05-24

国家自然科学基金(编号: 21571115, 21641011)、福建省自然科学基金(编号: 2015J01053)、山东省自然科学基金(编号: ZR2014BM027)、 山东大学青年学者未来计划(编号: 2015WLJH24)和福建省高校新世纪人才计划资助项目

\begin{abstract}
摘要银硫纳米簇合物的形成, 因涉及多个组分的组装过程, 其合成富有挑战性. 而阴离子模板法是一种 简单有效的合成银硫纳米簇合物的方法,特别是在对银硫纳米簇合物结构的控制方面表现出独特的优越性. 结合本课题的工作, 我们综述了简单的球形阴离子(如卤素离子、 $\mathrm{S}^{2-}$ 等), 三角形阴离子(如 $\mathrm{CO}_{3}{ }^{2-} 、 \mathrm{NO}_{3}{ }^{-}$等)及 复杂的多酸阴离子(如 $\left[\mathrm{W}_{6} \mathrm{O}_{21}\right]^{6-} 、 \mathrm{Mo}_{6} \mathrm{O}_{22}{ }^{8-}$ 等) 为模板剂合成银硫纳米簇合物的研究进展, 同时探索了合成的 银硫纳米簇合物的结构和发光性能.
\end{abstract}

关键词银硫纳米簇合物, 晶体结构, 阴离子模板, 发光性能

\section{1 引言}

银离子可以呈现多种氧化态, 其中 1 价银的配位 数可以从二配位到八配位变化. 几何上邻近的 1 价银 离子由于 $5 \mathrm{~s}$ 和 $5 \mathrm{p}$ 轨道能级接近, 可以形成银银弱作用, 即亲银作用. 通过亲银作用所形成的含银簇合物由 于具有新颖的结构和有趣的性能而备受关注, 而炔基 $\mathrm{RC} \equiv \mathrm{C}$ - 是构筑含银簇合物中重要的有机配体之一, 由 其所组装形成的炔银簇合物自1960年由Nast等报道后 至今仍广泛研究 ${ }^{[1,2]}$.

硫醇和炔配体与银成键时呈现类似的配位模式, 而且硫原子属于软碱, 根据软硬酸碱理论, 其与银离
子成键能力极强, 成键类型繁多, 不仅可以用 $\mathrm{s} 、 \mathrm{p}$ 轨 道与金属成键, 而且配位几何构型多种多样. 所以人 们希望用硫醇配体替代炔基配体来构筑银硫族合物. 这不仅对于丰富银簇合物的结构化学具有重要的理 论意义, 而且在生命科学及材料科学等诸多领域都具 有潜在的应用价值. 近些年, 随着实验技术和测试手 段的发展, 一系列银硫族合物被合成出来, 并用 $\mathrm{X}$ 射线 单晶衍射进行了结构表征. 其中如Jin、Fenske、Vilar 等国外课题组和香港中文大学、厦门大学、山东大 学、东北师范大学、安徽大学、郑州大学等国内许 多研究机构都取得了一定的进展 ${ }^{[3,4]}$. 尽管如此, 由于 配体、溶剂、合成条件等因素对银硫簇合物的结构

引用格式: 骆耿耿, 王芝, 程丽萍, 赵全芹, 王兴坡, 孙頔. 银硫纳米簇合物的阴离子模板法合成、结构与发光性能. 中国科学: 化学, 2017, 47: 695-704 Luo G, Wang Z, Cheng LP, Zhao QQ, Wang XP, Sun D. Synthesis, structures and luminescence of silver (I) thiolate nanoclusters based on anion templates. Sci Sin Chim, 2017, 47: 695-704, doi: 10.1360/N032016-00238 
影响比较大, 在银硫族合物的合成中还没有建立完善 成熟的规律, 对材料设计和合成的过程难以完全掌握 和控制. 因此, 积累丰富的合成素材和探索合成规律 显得尤其重要. 结合本课题组近几年内在银硫纳米簇 合物方面的工作, 本文对银硫簇合物的合成、结构和 发光性能进行了总结, 重点集中在仅含有硫醇配体、 晶体结构表征明确、分子尺寸大小在纳米级的银硫 簇合物上.

\section{2 银硫纳米簇合物的合成和结构}

\section{1 银硫纳米簇合物的无模板合成}

2004 2009 年, Fenske 课 题组 ${ }^{[5]}$ 在银硫纳米簇 合物的合成上做了一些先驱性的工作。他们利用 叔丁基硫醇银与含 $-\mathrm{SiMe}_{3}$ 基团的高活性硫源 (如 $\left.\mathrm{S}\left(\mathrm{SiMe}_{3}\right)_{2} 、 \mathrm{RSSiMe}_{3}\right)$ 在低温下反应合成了一系列高核 银硫簇合物, 其中核数超过 100 的银硫纳米簇合物有: $\mathrm{Ag}_{123} \mathrm{~S}_{35}\left(\mathrm{~S}^{t} \mathrm{Bu}\right)_{50} 、 \mathrm{Ag}_{188} \mathrm{~S}_{124}\left(\mathrm{~S}^{t} \mathrm{Bu}\right)_{96} 、 \mathrm{Ag}_{352} \mathrm{~S}_{128}\left(\mathrm{~S}^{t} \mathrm{C}_{5} \mathrm{H}_{11}\right)$ 和 $\mathrm{Ag}_{490} \mathrm{~S}_{188}\left(\mathrm{~S}^{t} \mathrm{C}_{5} \mathrm{H}_{11}\right)_{114}$, 而 $\mathrm{Ag}_{490} \mathrm{~S}_{188}\left(\mathrm{~S}^{t} \mathrm{C}_{5} \mathrm{H}_{11}\right)_{114}$ 是目前核 数最高的银硫簇合物. 但是Fenske 等 ${ }^{[5]}$ 所报道的这些 高核银硫纳米簇合物的结构杂乱无章, 不仅没有结构 美学和对称性可言, 而且离簇中心越近的银原子和硫 原子, 无序程度越高, 晶体结构根本不能精确确定, 同 时也没有相关发光性能报道, 无法深入探索银硫纳米 簇合物结构与发光性能之间的构-效关系.

2010 年, 厦门大学王泉明课题组 ${ }^{[6]}$ 将 $\mathrm{AgBF}_{4}$ 、 $\mathrm{NH}_{2} \mathrm{NH}_{2}$ 和 $\mathrm{AgSBu}^{t}$ 加入到甲醇中, $65^{\circ} \mathrm{C}$ 加热反 应后得到一个高对称性的银硫纳米簇合物 $\left[\mathrm{Ag}_{62} \mathrm{~S}_{13}\left(\mathrm{SBu}^{t}\right)_{32}\right]\left(\mathrm{BF}_{4}\right)_{4}$. 单晶结构分析表明, 该簇合 物是一个具有核-壳结构的阳离子簇(图1(a)), 包含 $\mathrm{Ag}_{14} \mathrm{~S}_{13}$ 核层和 $\mathrm{Ag}_{48}\left(\mathrm{SBu}^{t}\right)_{32}$ 壳层(图1(b, c)), 核的最中间 为一个 $\mathrm{S}^{2-}$, 被 8 个 $\mathrm{Ag}^{+}$构成的立方体包裹着, 其余 6 个 $\mathrm{Ag}^{+}$分别位于立方体的 6 个面上, 剩下的 12 个 $\mathrm{S}^{2-}$ 位于 立方体的 12 条边上.

山东大学孙頔课题组 ${ }^{[7]}$ 选用链状结构的阴离子 银聚合物 $\left\{\left[\mathrm{HNEt}_{3}\right]_{2}\left[\mathrm{Ag}_{10}\left(\mathrm{SC}_{6} \mathrm{H}_{4} \mathrm{Bu}^{t}\right)_{12}\right]_{n}\right.$ 为反应原料和 $\mathrm{CF}_{3} \mathrm{COOAg}$ 通过一锅法合成了一个 37 核银硫簇合物 $\left\{\left(\mathrm{HNEt}_{3}\right)\left[\mathrm{Ag}_{37} \mathrm{~S}_{4}\left(\mathrm{SC}_{6} \mathrm{H}_{4} \mathrm{Bu}^{t}\right)_{24}\left(\mathrm{CF}_{3} \mathrm{COO}\right)_{6}\left(\mathrm{H}_{2} \mathrm{O}\right)_{12}\right]\right\}$. 该簇 合物是一个阴离子簇, 内部是一个 $\mathrm{AgS}_{4}$ 构成的四面体, 外围 $\mathrm{Ag}_{36}$ 壳上加盖着 24 个 $\mu_{4}{ }^{-}{ }^{t} \mathrm{BuC}_{6} \mathrm{H}_{4} \mathrm{~S}^{-}, 6$ 个 $\mu_{2}-\mathrm{CF}_{3} \mathrm{COO}^{-}$ 和 12 个 $\mathrm{H}_{2} \mathrm{O}$ (图2(a)). 整个阴离子簇是一个高对称性的 球形结构, 直径约为 $2.2 \mathrm{~nm}$ (图2(b)). 其外围由 36 个 $\mathrm{Ag}^{+}$ 696 (a)

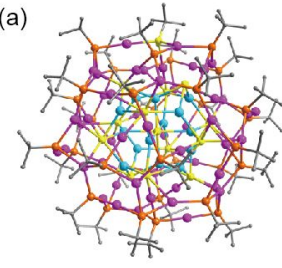

(b)

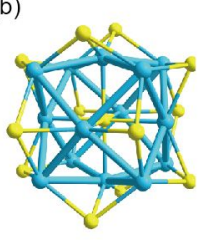

(c)

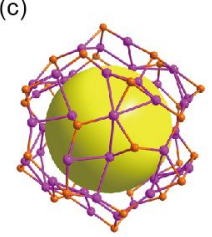

图 1 银硫纳米簇合物 $\left[\mathrm{Ag}_{62} \mathrm{~S}_{13}\left(\mathrm{SBu}^{\dagger}\right)_{32}\right]\left(\mathrm{BF}_{4}\right)_{4}$. (a) $\left[\mathrm{Ag}_{62} \mathrm{~S}_{13}-\right.$ $\left.\left(\mathrm{SBu}^{t}\right)_{32}\right]^{4+}$ 阳离子簇的对称结构; (b) $\mathrm{Ag}_{14} \mathrm{~S}_{13}$ 核结构; (c) $\mathrm{Ag}_{48}\left(\mathrm{SBu}^{t}\right)_{32}$ 壳结构(网络版彩图) (a)

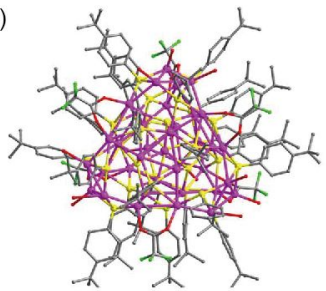

(c)

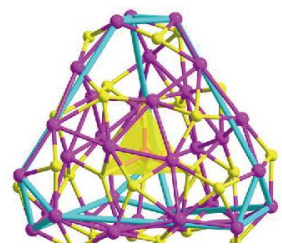

(b)

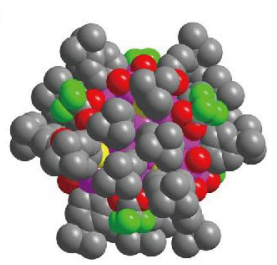

(d)

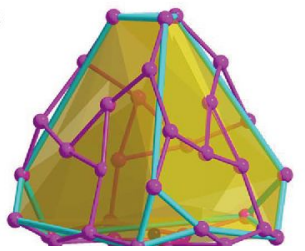

图 $2\left[\mathrm{Ag}_{37} \mathrm{~S}_{4}\left(\mathrm{SC}_{6} \mathrm{H}_{4}{ }^{t} \mathrm{Bu}\right)_{24}\left(\mathrm{CF}_{3} \mathrm{COO}\right)_{6}\left(\mathrm{H}_{2} \mathrm{O}\right)_{12}\right]^{-}$. (a) 球棍结构 图; (b) 分子填充图; (c) $\mathrm{Ag}_{36}$ 构成的阿基米德多面体外壳和 $\mathrm{AgS}_{4}$ 构成的柏拉图多面体内核; (d) $\mathrm{Ag}_{36}$ 在外壳上的构成图 (网络版彩图)

通过 $\mathrm{Ag} \cdots \mathrm{Ag}$ 作用连接构成, $\mathrm{Ag}_{36}$ 外壳是由 4 个六边形 和 4 个三角形构成, 其中的 18 条边和 12 个顶点可以构 成13个阿基米德多面体(阿基米德多面体是使用两种 或两种以上共边的正多边形为面的凸多面体)中最简 单的一个, 即截角四面体, 如图2(c)所示. 截角四面体 顶上的每个三角形是由 3 个 $\mathrm{Ag}^{+}$通过 $\mathrm{Ag} \cdots \mathrm{Ag}$ 作用构成, 它的六边形是由 12 个 $\mathrm{Ag}^{+}$通过 $\mathrm{Ag} \cdots \mathrm{Ag}$ 作用形成. 24 个 $^{t} \mathrm{BuC}_{6} \mathrm{H}_{4} \mathrm{~S}^{-}$配体与六边形表面的 $\mathrm{Ag}$ 离子形成四配位. $\mu_{2}-\mathrm{CF}_{3} \mathrm{COO}^{-}$和水分子连接在截角四面体的边上共同 来完成整个结构.

阴离子簇的内核是由 $\mathrm{Ag}^{+}$与 4 个对称产生的 $\mathrm{S}^{2-}$ 配 位构成的一个柏拉图四面体结构. 值得一提的是, $\mathrm{S}^{2-}$ 并不是作为反应物加入的, 而是在溶剂热合成条件下， 对叔丁基苯硫酚中的 $\mathrm{C}-\mathrm{S}$ 原位断裂产生的, 这种现象 在其他的相关银簇的报道中也可以看到. 这对于形成 大的银族非常必要, 因为它在簇中可以连接多个金属 
离子. 如果反应原料直接加入 $\mathrm{S}^{2-}$ 则容易形成 $\mathrm{Ag}_{2} \mathrm{~S}$ 沉 淀. 内核的 4 个 $\mathrm{S}^{2-}$ 为四配位, 与外围硫醇的硫原子配位 模式不同. 包围的 $\mathrm{AgS}_{4}$ 四面体支撑着整个簇的外围结 构, 有利于稳定整个阴离子簇的结构.

在拓扑简化下, 整个阴离子簇的结构就像一个笼 子, 内嵌柏拉图多面体, 外围由阿基米德多面体包围, 即Keplerate型(图3 $)^{[8]}$. 据我们所知, 该阴离子簇是第一 个由柏拉图多面体为模板的阿基米德多面体. 它的形 成是阿基米德和柏拉图多面体之间的几何关系的一 个表现.

\section{2 银硫纳米簇合物的阴离子模板合成}

Busch对于化学模板的定义是: 能够通过调控原 子间的组装, 使原子间形成特定连接而构成一种或 多种几何构型的化学物质称为化学模板 ${ }^{[9]}$. 根据带电 荷情况, 化学模板通常可分为阳离子模板和阴离子模 板. 阳离子作为模板控制反应的研究已经有几十年 的历史了, 其中最具有代表的是冠醚、穴状化合物和 金属酞菁的合成. 相对而言, 阴离子的分散能、 $\mathrm{pH}$ 敏 感性和相对高的溶剂化自由能等性质 ${ }^{[10]}$, 限制了其作 为模板的发展. 然而, 阴离子具有以下特点: (1) 半径 较大, 电子云密度较低; (2) 尺寸多样化, 具有不同的 几何构型, 如球形 $\left(\mathrm{F}^{-} 、 \mathrm{Cl}^{-} 、 \mathrm{Br}^{-} 、 \mathrm{I}^{-} 、 \mathrm{~S}^{2-}\right)$ 、平面三角 形 $\left(\mathrm{NO}_{3}{ }^{-} 、 \mathrm{CO}_{3}{ }^{2-}\right)$ 、四面体型 $\left(\mathrm{ClO}_{4}{ }^{-} 、 \mathrm{CrO}_{4}{ }^{2-} 、 \mathrm{SO}_{4}{ }^{2-}\right.$ 、 $\left.\mathrm{PO}_{4}{ }^{3-}\right)$ 等, 使其作为模板指导银簇合物的合成获得越 来越多的关注.

第一个以阴离子为模板合成的银簇合物可追溯 到2001年, Mingos 课题组 ${ }^{[11]}$ 在合成二索烃金的反应 中, 意外得到了以氯离子为模板的笼状炔银簇合物 $\left[\mathrm{Ag}_{14}\left(\mathrm{C} \equiv \mathrm{CBu}^{\dagger}\right)_{12} \mathrm{Cl}\right] \mathrm{OH}$. 该簇合物结构为 14 个 $\mathrm{Ag}^{+}$包裹 球状 $\mathrm{Cl}^{-}$组成了近似规则的十二面体. 反应过程中最 初的卤素来源于溶剂氯仿脱下来的氯离子, 在随后的 实验中, 他们分别用 $\left[\mathrm{NMe}_{4}\right] \mathrm{Cl} 、\left[\mathrm{NBu}_{4}\right] \mathrm{Br} 、\left[\mathrm{NBu}_{4}\right] \mathrm{F}$ 提 供模板阴离子, 得到了同构的簇合物 ${ }^{[12]}$. 这证明了阴 离子模板在笼状炔银簇合物的形成中的显著作用. 该 课题组的这两篇报道开辟了阴离子模板法合成炔银 簇合物的新方法, 对后来者的工作有很好的启发作用. 相对于阴离子模板法在炔银化合物研究中的蓬勃发 展, 该方法应用于银硫簇合物的报道较为少见. 考虑 到硫醇银配体和炔银配体具有相似的结构, 而且硫原 子具有较强的配位能力, 与银的配位具有多种方式, 易成簇. 本课题组近年来模仿阴离子模板法指导合成

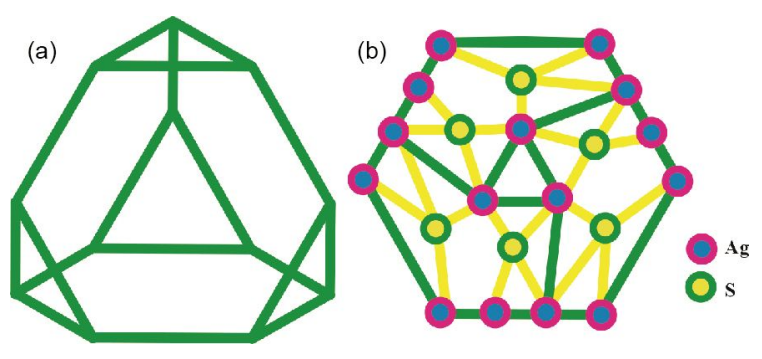

图 3 阿基米德多面体结构图(a)和每个六边形面上的银和 硫原子的分布(b) (网络版彩图)

炔银簇合物的方法来进行银硫簇合物的合成, 取得了 一些进展. 以下将结合课题组的研究工作总结简单的 球状阴离子, 三角形阴离子及复杂的多酸阴离子为模 板剂合成银硫纳米簇合物的进展.

\subsection{1 简单阴离子模板构筑的银硫纳米簇合物}

2010 年, $\mathrm{Liu}$ 等 ${ }^{[13]}$ 采用配体 $\left[\mathrm{S}_{2} \mathrm{P}(\mathrm{OEt})_{2}\right]^{-}$合成了一 维链状聚合物 $\left[\mathrm{Ag}_{5}\left\{\mathrm{~S}_{2} \mathrm{P}(\mathrm{OEt})_{2}\right\}_{4}\left(\mathrm{PF}_{6}\right)_{n}\right.$, 并以其为前驱 体, 当加入适当的卤素阴离子 $\left(\mathrm{F}^{-} 、 \mathrm{Cl}^{-}\right)$后, 发现该 聚合物可以转化为以 $\mathrm{F}^{-} 、 \mathrm{Cl}^{-}$为模板的银硫簇合物 $\left[\mathrm{Ag}_{8}(\mathrm{X})\left\{\mathrm{S}_{2} \mathrm{P}(\mathrm{OEt})_{2}\right\}_{6}\right]\left(\mathrm{PF}_{6}\right)(\mathrm{X}=\mathrm{F} 、 \mathrm{Cl})$, 该簇合物结构中 8 个银离子组成变形的立方体将卤素离子包裹. 这是 首例报道的阴离子模板法合成的银硫簇合物.

受其启发, 2010年, 厦门大学郑南峰课题组 ${ }^{[14]}$ 以 链状结构的阴离子银聚合物 $\left\{\left[\mathrm{HNEt}_{3}\right]_{2}\left[\mathrm{Ag}_{10}\left(\mathrm{SC}_{6} \mathrm{H}_{4}{ }^{t} \mathrm{Bu}-\right.\right.\right.$ $\left.\left.4)_{12}\right]\right\}_{n}$ 为原料, 与 $\left(\mathrm{C}_{4} \mathrm{H}_{9}\right)_{4} \mathrm{NBr}$ 加热反应制备了一个 $\mathrm{Br}^{-}$诱导合成的具有高对称性的 36 核银硫簇合物 $\left[\mathrm{Br} @ \mathrm{Ag}_{36}\left(\mathrm{SC}_{6} \mathrm{H}_{4}{ }^{t} \mathrm{Bu}\right)_{36}\right]^{-}$. 在该阴离子簇合物结构中, $\mathrm{Br}^{-}$位于簇中心, 与在其上下的两个 $\mathrm{Ag}_{3} \mathrm{~S}_{3}$ 单元中的银 配位形成三明治形的核心单元 $\mathrm{Br}^{-} @ \mathrm{Ag}_{18} \mathrm{~S}_{12}$, 该单元被 $\mathrm{Ag}_{12} \mathrm{~S}_{6}$ 环包围, 在外面还有 $\mathrm{Ag}_{12} \mathrm{~S}_{12}$ 环将其包围, 环与环 之间通过 $\mathrm{Ag}-\mathrm{S}$ 键连接在一起形成碟状簇合物.

2012年, 东北师范大学苏忠民课题组 ${ }^{[15]}$ 使用 ${ }^{t} \mathrm{BuS}^{-}$ 配体及辅助配体 $\mathrm{CF}_{3} \mathrm{COO}^{-}$, 合成了含有 $\mathrm{Cl}^{-}$为模板的 20 核银簇 $\left[\mathrm{Ag}_{20}\left(\mathrm{SBu}^{\dagger}\right)_{10}\left(\mathrm{CF}_{3} \mathrm{COO}\right)_{2}\right] \mathrm{Cl} \cdot\left(\mathrm{CF}_{3} \mathrm{COO}\right)_{7} \cdot 5 \mathrm{CH}_{3} \mathrm{OH}$ (简称 $\mathrm{Ag}_{20} \mathrm{~S}_{10}$, 图4). 该 $\mathrm{Ag}_{20} \mathrm{~S}_{10}$ 簇具有 3 层平行的代表性 大环: 中间的 $\mathrm{Ag}_{10}$ 环和在其上下两层倒置的 $\mathrm{Ag}_{5} \mathrm{~S}_{5}$ 五 角星, 整个 $\mathrm{Ag}_{20} \mathrm{~S}_{10}$ 族可以看作一个夹心结构. 桥连的 ${ }^{t} \mathrm{BuS}^{-}$配体和支撑的 $\mathrm{CF}_{3} \mathrm{COO}^{-}$辅助配体以交替的形式 处于 $\mathrm{Ag}_{10}$ 环的上下两侧, 其中 $\mathrm{Ag}_{10}$ 环由 10 个 $\mathrm{Ag}^{+}$通过亲 银作用连接而成. 该 $\mathrm{Ag}_{20} \mathrm{~S}_{10}$ 簇的外围直径 $10.2 \AA$, 厚 度 $4.2 \AA$, 除了中心的 $\mathrm{Cl}^{-}$外具有一个完全的空腔, 这表 


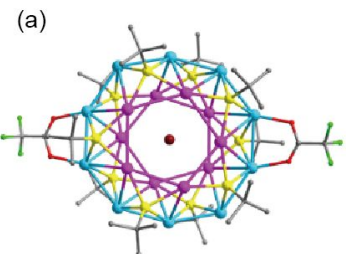

(b)

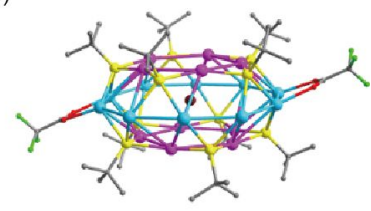

图 4 (a) $\mathrm{Ag}_{20} \mathrm{~S}_{10}$ 簇中夹心结构的标准球棒模型图; (b) $\mathrm{Ag}_{20} \mathrm{~S}_{10}$ 簇的球棒模型的侧视图(所有的氢原子已省略) (网络版彩图)

明 $\mathrm{Cl}^{-}$是作为一个阴离子模板诱导纳米级大环结构的 形成.

苏忠民等 ${ }^{[16]}$ 为了制备由 $\left[\mathrm{CrMo}_{6} \mathrm{O}_{24} \mathrm{H}_{6}\right]^{3-}$ 多阴离 子诱导形成的高核银硫簇合物, 尝试通过 $\mathrm{AgSBu}^{t}$ 与 $\left(\mathrm{NH}_{4}\right)_{3}\left[\mathrm{CrMo}_{6} \mathrm{O}_{24} \mathrm{H}_{6}\right] \cdot 7 \mathrm{H}_{2} \mathrm{O} 、 \mathrm{Ni}\left(\mathrm{CH}_{3} \mathrm{COO}\right)_{2} \cdot 4 \mathrm{H}_{2} \mathrm{O}$ 、 $\mathrm{AgCF}_{3} \mathrm{CO}_{2}$ 和 $\mathrm{AgBF}_{4}$ 在甲醇 - 乙腈 $-N, N-$ 二甲基甲 酰胺 (DMF) 混合溶液中自组装, 意外地获得了 一个一维三明治型的银硫纳米簇合物 $\left[\left(\mathrm{CO}_{3}{ }^{2-}\right) @\right.$ $\left.\left.\mathrm{Ag}_{20}\left(\mathrm{SBu}^{t}\right)_{10}\left(\mathrm{CH}_{3} \mathrm{CO}_{2}\right)_{8}(\mathrm{DMF})_{2}\right] \cdot 2 \mathrm{H}_{2} \mathrm{O}\right\}_{n}$. 这里, $\mathrm{CO}_{3}{ }^{2-}$ 作 为一个阴离子模板被封装在内部用来诱导形成具有 大环基夹心结构 $\left[\mathrm{Ag}_{20}\left(\mathrm{SBu}^{\dagger}\right)_{10}\left(\mathrm{CH}_{3} \mathrm{CO}_{2}\right)_{8}(\mathrm{DMF})_{2}\right]^{2+}$ 阳 离子簇(图5). 由于起始反应物中并没有引入 $\mathrm{CO}_{3}{ }^{2-}$, 而 且在室温下 $\mathrm{DMF}$ 分解产生 $\mathrm{CO}_{3}{ }^{2-}$ 也是不可能的, 因此 推测该簇合物中出现的 $\mathrm{CO}_{3}{ }^{2-}$ 应该是在晶体培养过 程中, 空气中的 $\mathrm{CO}_{2}$ 在包含 DMF溶液 $(\mathrm{pH}=7.5)$ 中转化 形成的. 因此, DMF在该簇合物的组装过程不仅可 以作为一种弱碱来协助 $\mathrm{CO}_{2}$ 经水合作用转化为 $\mathrm{CO}_{3}{ }^{2-}$, 还可以作为辅助配体与银离子配位来稳定最终的簇 结构.

2013 年, 孙頔课题 组 ${ }^{[17]}$ 通过 $\mathrm{AgSBu}^{t}$ 和 $\mathrm{AgNO}_{3}$ 在甲醇 - 乙醇-DMF混合溶液中通过超声反应, 分 离出 $\mathrm{CO}_{3}{ }^{2-}$ 为模板所诱导形成的 20 核银硫簇合物 $\left[\left(\mathrm{CO}_{3}{ }^{2-}\right) @ \mathrm{Ag}_{20}\left(\mathrm{SBu}^{\dagger}\right)_{10}(\mathrm{DMF})_{6}\left(\mathrm{NO}_{3}\right)_{8}\right]$ (图6). 该簇合物 包含了由 2 个五角星形的 $\mathrm{Ag}_{5} \mathrm{~S}_{5}$ 和 1 个 $\mathrm{Ag}_{10}$ 环所构成的 类鼓状结构 $\mathrm{Ag}_{20} \mathrm{~S}_{10} . \mathrm{Ag}_{20} \mathrm{~S}_{10}$ 外围直径 $10 \AA$, 厚度 $4 \AA$. $\mathrm{CO}_{3}{ }^{2-}$ 处于 $\mathrm{Ag}_{20} \mathrm{~S}_{10}$ 簇合物的中心, 通过 $\mu_{6}-\eta^{2}: \eta^{2}: \eta^{2}$ 模式连 接外围的银离子. 这结构与苏忠民等 ${ }^{[16]}$ 研究的类似, 而且由于起始反应物中并没有引入 $\mathrm{CO}_{3}{ }^{2-}$, 推测簇合物 中出现的 $\mathrm{CO}_{3}{ }^{2-}$ 应该是通过固定大气中的 $\mathrm{CO}_{2}$ 得到的.

有趣的是, 当尝试把反应原料中的 $\mathrm{AgNO}_{3}$ 改为 $\mathrm{AgOAc}$ 后, 与 $\mathrm{A} \mathrm{gSBu}^{t}$ 一起加入到甲醇-乙醇-DMF 混合溶剂中超声反应, 室温下缓慢挥发溶液同时得 到了一个 $\mathrm{S}^{2-}$ 为模板诱导形成的 56 核银硫纳米簇合物

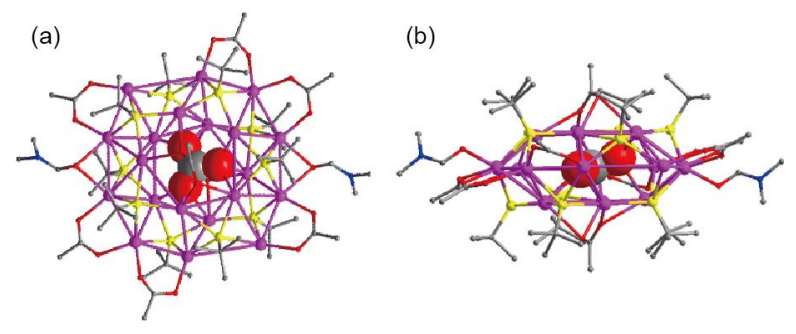

图 5 (a) $\left[\left(\mathrm{CO}_{3}{ }^{2-}\right) @ \mathrm{Ag}_{20}\left(\mathrm{SBu}^{\dagger}\right)_{10}\left(\mathrm{CH}_{3} \mathrm{CO}_{2}\right)_{8}(\mathrm{DMF})_{2}\right]$ 簇的球棒 模型. 俯视图(a)和侧视图(b) (所有的氢原子已省略) (网络 版彩图)

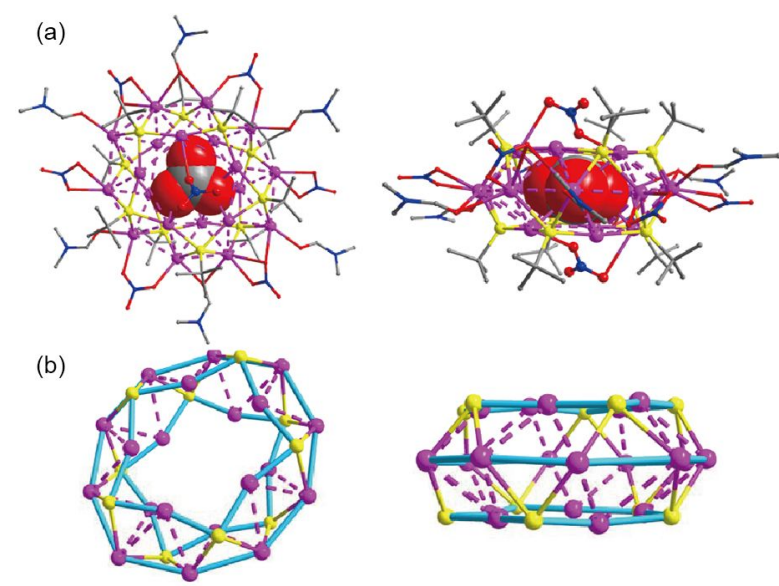

图 6 (a) $\left[\left(\mathrm{CO}_{3}{ }^{2-}\right) @ \mathrm{Ag}_{20}\left(\mathrm{SBu}^{t}\right)_{10}(\mathrm{DMF})_{6}\left(\mathrm{NO}_{3}\right)_{8}\right]$ 簇合物标准球 棒模型的俯视图(左)和侧视图(右) (所有的氢原子已省略); (b) 2 个五角星形的 $\mathrm{Ag}_{5} \mathrm{~S}_{5}$ 和 1 个 $\mathrm{Ag}_{10}$ 环所构成的类鼓状结构 $\mathrm{Ag}_{20} \mathrm{~S}_{10}$ (网络版彩图)

$\left[\left(\mathrm{S}^{2-}\right) @ \mathrm{Ag}_{56} \mathrm{~S}_{12}\left(\mathrm{SBu}^{t}\right)_{20}\right]\left(10 \mathrm{OAc} \cdot 6 \mathrm{DMF} \cdot \mathrm{CH}_{3} \mathrm{OH}\right)$ 和 $\mathrm{CO}_{3}{ }^{2-}$ 为模板诱导形成的 20 核银硫簇合物 $\left[\left(\mathrm{CO}_{3}{ }^{2-}\right) @\right.$ $\left.\mathrm{Ag}_{20}\left(\mathrm{SBu}^{t}\right)_{10}(\mathrm{OAc})_{8}(\mathrm{DMF})_{4}\right]\left(\mathrm{DMF} \cdot \mathrm{CH}_{3} \mathrm{OH}\right) \quad\left(\right.$ 图 7) ${ }^{[18]}$, 其中 $\mathrm{S}^{2-}$ 产生于 ${ }^{t} \mathrm{BuS}^{-}$中 $\mathrm{C}-\mathrm{S}$ 键的原位断裂, 而 $\mathrm{CO}_{3}{ }^{2-}$ 则来自空气中 $\mathrm{CO}_{2}$ 的转化. 红色晶体 $\left[\left(\mathrm{S}^{2-}\right) @\right.$ $\left.\mathrm{Ag}_{56} \mathrm{~S}_{12}\left(\mathrm{SBu}^{t}\right)_{20}\right]\left(10 \mathrm{OAc} \cdot 6 \mathrm{DMF} \cdot \mathrm{CH}_{3} \mathrm{OH}\right)$ 为球形簇合物, 分别由 56 个 $\mathrm{Ag}^{+} 、 20$ 个 $^{t} \mathrm{BuS}^{-}$配体和 12 个 $\mathrm{S}^{2-}$ 组成. 它 可以被看作包含内部的 $\mathrm{Ag}_{14}$ 和外部的 $\mathrm{Ag}_{42}\left(\mathrm{SBu}^{\dagger}\right)_{20}$ 的核壳结构. 8 个 $\mathrm{Ag}^{+}$形成的立方体, 额外的 6 个 $\mathrm{Ag}^{+}$ 通过 $\mathrm{Ag} \cdots \mathrm{Ag}$ 作用覆盖在 6 个矩形面中, 形成有趣的 六包裹立方构型的多面体。中心 $\mathrm{S}^{2-}$ 离子采用线型 配位模式,而原位产生的 12 个 $\mathrm{S}^{2-}$ 离子嵌在内部六 包裹立方和外部 $\mathrm{Ag}_{42}\left(\mathrm{SBu}^{t}\right)_{20}$ 壳之间的空隙中. $\mathrm{S}^{2-}$ 离子和 $\mathrm{Ag} \cdots \mathrm{Ag}$ 作用连接着核壳形成整个纳米簇。 $\left[\left(\mathrm{CO}_{3}{ }^{2-}\right) @ \mathrm{Ag}_{20}\left(\mathrm{SBu}^{\dagger}\right)_{10}(\mathrm{OAc})_{8}(\mathrm{DMF})_{4}\right]\left(\mathrm{DMF} \cdot \mathrm{CH}_{3} \mathrm{OH}\right)$ 为 
(a)

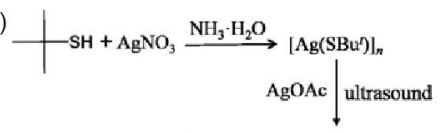

$\left[\left(\mathrm{S}^{2}\right) @ \mathrm{Ag}_{8_{86}} \mathrm{~S}_{12}\left(\mathrm{SBu}_{200}\right]\left(1 \cdot 100 \mathrm{Ac} \cdot 6 \mathrm{DMF} \cdot \mathrm{CH}_{3} \mathrm{OH}\right)\right.$ $\left[\left(\mathrm{CO}_{3}^{2}\right) @ \mathrm{Ag}_{20}(\mathrm{SBu})_{10}(\mathrm{OAc})_{8}(\mathrm{DMF})_{4}\right]\left(\mathbf{2} \cdot \mathrm{DMF} \cdot \mathrm{CH}_{3} \mathrm{OH}\right)$
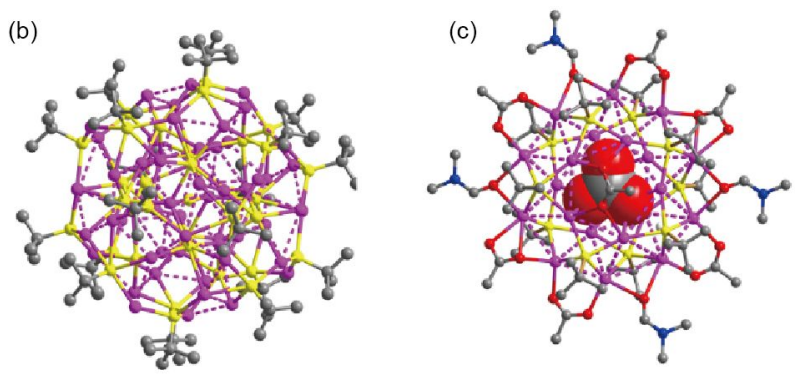

图 7 (a) 两类银硫纳米簇合物的溶液超声合成过程; (b) $\left[\left(\mathrm{S}^{2-}\right) @ \mathrm{Ag}_{56} \mathrm{~S}_{12}\left(\mathrm{SBu}^{t}\right)_{20}\right]\left(10 \mathrm{OAc} \cdot 6 \mathrm{DMF} \cdot \mathrm{CH}_{3} \mathrm{OH}\right)$ 的球棍结构 图; (c) $\left[\left(\mathrm{CO}_{3}{ }^{2-}\right) @ \mathrm{Ag}_{20}\left(\mathrm{SBu}^{\dagger}\right)_{10}(\mathrm{OAc})_{8}(\mathrm{DMF})_{4}\right]\left(\mathrm{DMF} \cdot \mathrm{CH}_{3} \mathrm{OH}\right)$ 的 球棍模型图(所有氢原子已省略) (网络版彩图)

白色晶体, 产率很低 $(<5 \%)$. 单晶结构分析表明, 其为圆

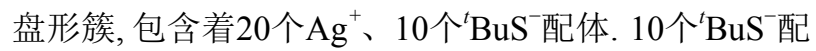
体采用 $\mu_{4}-\eta^{1}, \eta^{1}, \eta^{1}$ 配位模式, 连接 4 个 $\mathrm{Ag}^{+}$形成 10 个 $\mathrm{Ag}_{4} \mathrm{~S}$ 四方雉, 每个四方锥分别由 1 个矩形银底部和 4 个 $\mathrm{Ag}_{2} \mathrm{~S}$ 三角形面构成. 这 10 个 $\mathrm{Ag}_{4} \mathrm{~S}$ 四方雉共享他们的角形成 $\mathrm{Ag}_{20}$ 簇. 10 个银三角形进一步交替嵌入到每 3 个 $\mathrm{Ag}_{4} \mathrm{~S}$ 四方雉的空隙中. 8 个OAc 离子显示 3 种配位模式, 4 个 是 $\left(\kappa^{1}\right)-\left(\kappa^{1}\right)-\mu_{2}, 2$ 个是 $\left(\kappa^{2}\right)-\left(\kappa^{1}\right)-\mu_{3}$, 并与 $\mu_{3}$-DMF共同键合 外围的 $\mathrm{Ag}_{20} \mathrm{~S}_{10}$ 簇. $\mathrm{CO}_{3}{ }^{2-}$ 作为阴离子模板剂被包裹在簇 的中心, 并通过 $\left(\kappa^{3}\right)-\left(\kappa^{3}\right)-\left(\kappa^{3}\right)-\mu_{6}$ 模式连接外部的银簇.

2014年, 藏双全等 ${ }^{[19]}$ 通过 $\mathrm{AgS}^{t} \mathrm{Bu}$ 与 $\mathrm{AgCF}_{3} \mathrm{CO}_{2}$ 反 应, 在加入少量 $\mathrm{CS}_{2}$ 和 $\mathrm{AgNO}_{3}$ 下获得了一个33核银硫 簇 $\left[\mathrm{Ag}_{33} \mathrm{~S}_{3}\left(\mathrm{SBu}^{t}\right)_{16}\left(\mathrm{CF}_{3} \mathrm{CO}_{2}\right)_{9}\left(\mathrm{NO}_{3}\right)\left(\mathrm{CH}_{3} \mathrm{CN}\right)_{2}\right]\left(\mathrm{NO}_{3}\right)$ (图8). 该阳离子簇具有鸟巢形双层结构: 由 9 个银离子和 6 个 硫醇所构成内层 $\mathrm{Ag}_{9} \mathrm{~S}_{6}$ 中封包了一个 $\mathrm{NO}_{3}{ }^{-}$模板, 而外 层则由 $\mathrm{Ag}_{24} \mathrm{~S}_{10}$ 组成. 整个簇合物的内、外层之间通过 3 个 $\mathrm{S}^{2-}$ 和 $\mathrm{Ag} \cdots \mathrm{Ag}$ 亲银作用连接.

\subsection{2 复杂多酸阴离子模板构筑的银硫纳米簇 合物}

多酸是一类体积较大的含氧阴离子, 含有多个负 电荷, 可作为软配体与多个银原子配位, 其作为内嵌 阴离子被包裹在簇合物中心可以用来指导合成高核 数的银硫纳米簇合物.

2012年, 苏忠民等 ${ }^{[20]}$ 研究了一个以 $\mathrm{W}_{6} \mathrm{O}_{21}{ }^{6-}$ 多酸阴

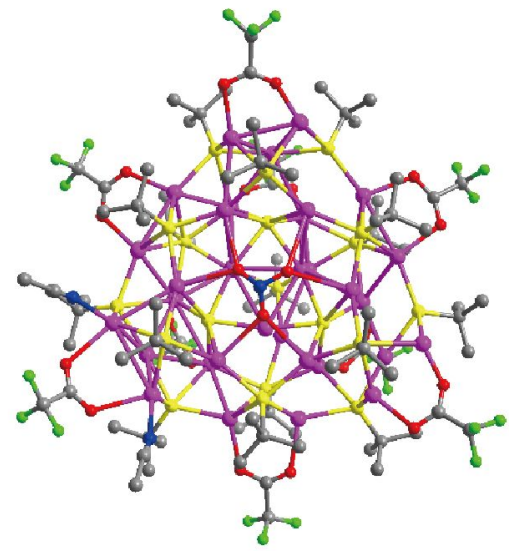

图 $8\left[\mathrm{Ag}_{33} \mathrm{~S}_{3}\left(\mathrm{SBu}^{t}\right)_{16}\left(\mathrm{CF}_{3} \mathrm{CO}_{2}\right)_{9}\left(\mathrm{NO}_{3}\right)\left(\mathrm{CH}_{3} \mathrm{CN}\right)_{2}\right]^{+}$阳离子簇的 晶体结构图(网络版彩图)

离子为模板所合成的一维银硫簇合物 $\left[\mathrm{Ag}_{34}\left(\mathrm{SBu}^{t}\right)_{26}\right.$ $\left.\left(\mathrm{W}_{6} \mathrm{O}_{21}\right)\left(\mathrm{CF}_{3} \mathrm{COO}\right)\right]\left(\mathrm{CF}_{3} \mathrm{COO}\right) \cdot \mathrm{Et}_{3} \mathrm{~N} \cdot 20 \mathrm{CH}_{3} \mathrm{OH}$. 具体合成 方法是将 $\mathrm{AgSBu}$ 溶于甲醇中, 加入三氟乙酸和三乙胺, 搅拌数分钟后, 加入 $\left({ }^{n} \mathrm{Bu}_{4} \mathrm{~N}\right)_{2}\left[\mathrm{~W}_{6} \mathrm{O}_{19}\right]$, 相应的灰紫色悬 浊液室温下搅拌 $55 \mathrm{~h}$ 后过滤, 滤液室温下缓慢挥发得 到亮黄色晶体. 单晶结构分析表明, 该簇合物的基本构 筑单元是一个纳米尺寸大小的核-壳结构, 分别由一个 $\left[\mathrm{W}_{6} \mathrm{O}_{19}\right]^{2-}$ 内核和一个 $\left[\mathrm{Ag}_{34}\left(\mathrm{SBu}^{\dagger}\right)_{26}\left(\mathrm{~W}_{6} \mathrm{O}_{21}\right)\left(\mathrm{CF}_{3} \mathrm{COO}\right)\right]^{7+}$ 阳离子簇外壳所组成, 如图 9 所示. 阳离子簇外壳上

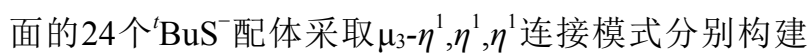
了 Ag-S 六边形、八边形、十边形、十二边形和十四 边形, 而另外两个 ${ }^{t} \mathrm{BuS}^{-}$配体则采取 $\mu_{2}-\eta^{1}, \eta^{1}$ 配位模式 构建了 $\mathrm{Ag}-\mathrm{S}$ 十六边形, 整个壳被 $\mathrm{Ag}-\mathrm{S}$ 键和 $\mathrm{Ag} \cdots \mathrm{Ag}$ 亲 银作用所稳定. $\mathrm{Ag}_{34}$ 笼足够容纳一个 $\left[\mathrm{W}_{6} \mathrm{O}_{21}\right]^{6-}$ 阴离子 (尺寸为 $7.5 \AA \times 8.1 \AA$ ). 其中, $\left[\mathrm{W}_{6} \mathrm{O}_{21}\right]^{6-}$ 阴离子由前驱体 $\left[\mathrm{W}_{6} \mathrm{O}_{19}\right]^{2-}$ 原位反应转变而成, 被阳离子银硫簇所包裹, 充当 $\mathrm{Ag}_{34}$ 的形成模板. $\left[\mathrm{W}_{6} \mathrm{O}_{21}\right]^{6}$ 结构是由 6 个 $\mathrm{WO}_{6}$ 正八 面体构成的, 外围附加的两个 $\mathrm{W}$ 原子以共边和共角的 模式依附到一个 $\mathrm{W}_{4} \mathrm{O}_{4}$ 立方烷型结构上. 价键计算证 实, $\left[\mathrm{W}_{6} \mathrm{O}_{21}\right]^{6-}$ 中的 $\mathrm{W}$ 原子是 +6 价, 说明在异构化的过程 中没有发生氧化还原反应. 整个簇合物通过 $\mathrm{Ag}-\mathrm{S}$ 键 作用沿 [100]方向进一步拓展为一维链状结构, 是第一 例桥连银簇和多阴离子的拓展结构.

2015年, 藏双全等 ${ }^{[2]}$ 研究了一个花生状的银硫 簇 $\left[\mathrm{Ag}_{62}\left(\mathrm{SBu}^{t}\right)_{40}\left(\mathrm{Mo}_{20} \mathrm{O}_{66}\right)\left(\mathrm{Mo}_{6} \mathrm{O}_{19}\right)_{3}\left(\mathrm{CH}_{3} \mathrm{CN}\right)_{2}\right] \cdot\left(\mathrm{CF}_{3} \mathrm{SO}_{3}\right)_{4}$ (图 10), 它由Lindquist型 $\left(\mathrm{Bu}_{4} \mathrm{~N}\right)_{2}\left[\mathrm{Mo}_{6} \mathrm{O}_{19}\right] 、 \mathrm{AgSBu}^{t}$ 、 $\mathrm{CF}_{3} \mathrm{SO}_{3} \mathrm{Ag}$ 和 $\mathrm{CF}_{3} \mathrm{SO}_{3} \mathrm{H}$ 在乙腈/甲醇混合溶剂中合成得 


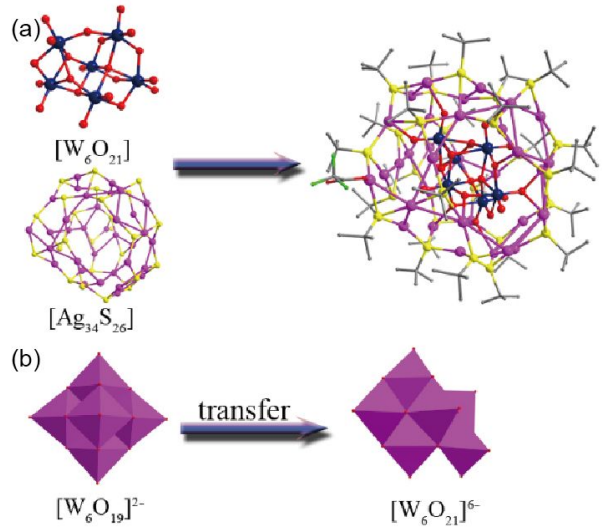

图 9 (a) 阳离子簇合物 $\left[\mathrm{Ag}_{34}\left(\mathrm{SBu}^{t}\right)_{26}\left(\mathrm{~W}_{6} \mathrm{O}_{21}\right)\left(\mathrm{CF}_{3} \mathrm{COO}\right)\right]^{7+}$ 的 核-壳结构(为了简便, 氢原子、 $\mathrm{CH}_{3} \mathrm{OH} 、 \mathrm{Et}_{3} \mathrm{~N}$ 和 $\mathrm{CF}_{3} \mathrm{COO}$-被 省略); (b) $\left[\mathrm{W}_{6} \mathrm{O}_{19}\right]^{2-}$ 异构化为 $\left[\mathrm{W}_{6} \mathrm{O}_{21}\right]^{6-}$ (网络版彩图)

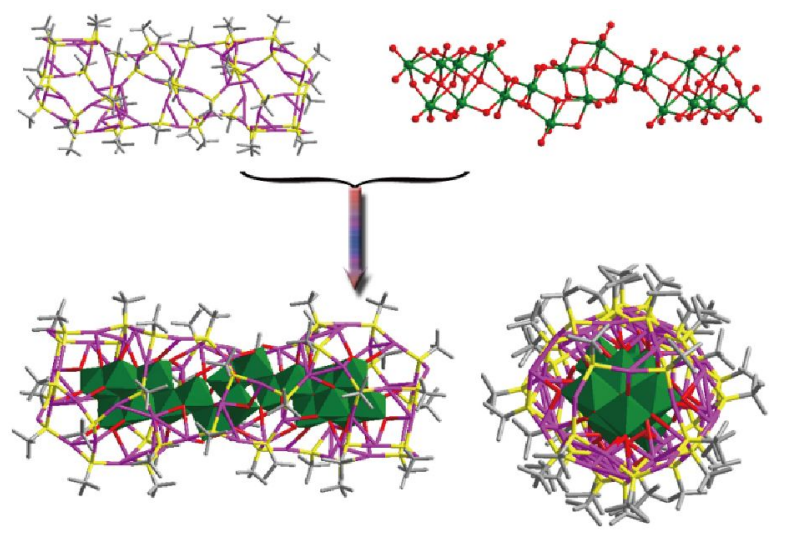

图 10 花生状的银硫纳米簇合物 $\left[\mathrm{Ag}_{62}\left(\mathrm{SBu}^{t}\right)_{40}\left(\mathrm{Mo}_{20} \mathrm{O}_{66}\right)-\right.$ $\left.\left(\mathrm{Mo}_{6} \mathrm{O}_{19}\right)_{3}\left(\mathrm{CH}_{3} \mathrm{CN}\right)_{2}\right] \cdot\left(\mathrm{CF}_{3} \mathrm{SO}_{3}\right)_{4}$ 的结构(网络版彩图)

到. 该簇合物由 $\left[\mathrm{Ag}_{62}\left(\mathrm{SBu}^{\dagger}\right)_{40}\right]^{22+}$ 外壳和 1 个 $\left[\mathrm{Mo}_{20} \mathrm{O}_{66}\right]^{12-}$ 多酸阴离子内壳所构成. $\left[\mathrm{Ag}_{62}\left(\mathrm{SBu}^{t}\right)_{40}\right]^{22+}$ 外壳留有足 够大的空腔 $(3.3 \mathrm{~nm} \times 1.7 \mathrm{~nm})$ 可以容纳 $\left[\mathrm{Mo}_{20} \mathrm{O}_{66}\right]^{12-}$ 离子 $(2.5 \mathrm{~nm} \times 0.8 \mathrm{~nm})$. $\left[\mathrm{Mo}_{20} \mathrm{O}_{66}\right]^{12-}$ 是由 Lindquist型前驱体 $\left[\mathrm{Mo}_{6} \mathrm{O}_{19}\right]^{2-}$ 原位反应形成的. 结构上, $\left[\mathrm{Mo}_{20} \mathrm{O}_{66}\right]^{12-}$ 可视 为由 $\left[\mathrm{Mo}_{6} \mathrm{O}_{18}\right]$ 桥联两个 $\left[\mathrm{Mo}_{7} \mathrm{O}_{24}\right]^{6-}$ 所构成. 与Lindquist 型前驱体 $\left[\mathrm{Mo}_{6} \mathrm{O}_{19}\right]^{2-}$ 相比, $\left[\mathrm{Mo}_{20} \mathrm{O}_{66}\right]^{12-}$ 具有更多的端基 氧及较高的电荷密度, 这不但可起到阴离子模板诱导 银硫簇的形成, 而且可与 $\mathrm{Ag}$ 形成额外的 $\mathrm{Ag}-\mathrm{O}$ 键来稳 定该簇合物。

2015年, 孙頔等 ${ }^{[22]}$ 以 $\left\{\left[\mathrm{HNEt}_{3}\right]_{2}\left[\mathrm{Ag}_{10}\left(\mathrm{SC}_{6} \mathrm{H}_{4} \mathrm{Bu}^{t}\right)_{12}\right]\right\}_{n}$ 为反应原料, 与 $\mathrm{CF}_{3} \mathrm{CO}_{2} \mathrm{Ag} 、\left(\mathrm{NH}_{4}\right)_{6} \mathrm{Mo}_{7} \mathrm{O}_{24}$ 在 溶剂 热合成反应下得到了一个 58 核银硫纳米簇合物
$\left[\mathrm{Mo}_{6} \mathrm{O}_{22} @ \mathrm{Ag}_{58} \mathrm{~S}_{2}\left(\mathrm{SC}_{6} \mathrm{H}_{4} \mathrm{Bu}^{t}\right)_{36}\left(\mathrm{CF}_{3} \mathrm{COO}\right)_{10}\left(\mathrm{H}_{2} \mathrm{O}\right)_{8}\right]$ (图11). $\mathrm{X}$ 射线单晶衍射表明, 该簇合物为核-壳结构, 包括 1 个 $\mathrm{Mo}_{6} \mathrm{O}_{22}{ }^{8-}$ 的多酸阴离子为内核和芒果型的 58 核银 硫簇(简写为 $\mathrm{Ag}_{58} \mathrm{~S}_{38}$ ) 为外壳. 芒果型的 $\mathrm{Ag}_{58} \mathrm{~S}_{38}$ 外壳可 以看作成 2 个 $\mathrm{Ag}_{10} \mathrm{~S}_{6}$ 的碗加盖在 $\mathrm{Ag}_{38} \mathrm{~S}_{26}$ 腰的上下两端. $\mathrm{Ag}_{58}$ 外壳上有 2 个由 ${ }^{t} \mathrm{BuC}_{6} \mathrm{H}_{4} \mathrm{~S}^{-}$配体中的 $\mathrm{C}-\mathrm{S}$ 断裂产生 的 $\mu_{2}-\mathrm{S}^{2-}$ 配位, 整个簇的中心为由 $\mathrm{Mo}_{7} \mathrm{O}_{24}{ }^{6-}$ 原位产生的 1 个 $\mathrm{Mo}_{6} \mathrm{O}_{22}{ }^{8-} . \mathrm{Mo}_{6} \mathrm{O}_{22}{ }^{8-}$ 内核连接着外围的 24 个银原子, 表明多酸表面高的电荷密度的氧原子可以吸引周围 的银离子形成 $\mathrm{Ag}-\mathrm{O}$ 键, 这也促进了外围 $\mathrm{Ag}-\mathrm{S}$ 键的形 成. 值得一提的是, 在温和的溶剂热条件下, 前驱体 $\mathrm{Mo}_{7} \mathrm{O}_{24}{ }^{6-}$ 原位转化为 $\mathrm{Mo}_{6} \mathrm{O}_{22}{ }^{8-}$. 据我们所知, 该簇合物 中的 $\mathrm{Mo}_{6} \mathrm{O}_{22}{ }^{8-}$ 在多酸化学中是罕见的, 目前报告的仅 有3例 ${ }^{[23-25]}$. 在 $\mathrm{Mo}_{6} \mathrm{O}_{22}{ }^{8-}$ 中, 6 个 $\mathrm{MoO}_{6}$ 正八面体通过共 边模式缩合在一起, 它既不同于传统的Lindquist型同 多酸 $\mathrm{Mo}_{6} \mathrm{O}_{19}{ }^{2-[26]}$, 也与其他的 $\mathrm{Mo}_{6} \mathrm{O}_{22}{ }^{8-}$ 不同, 这个是一 个 $\mathrm{Mo}_{4} \mathrm{O}_{4}$ 与另外的两个 $\mathrm{Mo}$ 原子相连, 形成一个二缺 位的同八钿酸 ${ }^{27]}$.

在超声条件下将该簇合物分散在乙醇中, 利用高 分辨透射电子显微镜观察其形貌. 图12表明该簇合物 以单分散颗粒的形式存在, 颗粒大小约为 $3.0 \mathrm{~nm}$, 这与 $\mathrm{X}$ 射线结构分析得到的簇合物的分子尺寸大小相当(最 大的 $\mathrm{C} \cdots \mathrm{C}$ 的距离为 $2.90 \mathrm{~nm}$ ). 一般来说, 多金属氧酸盐
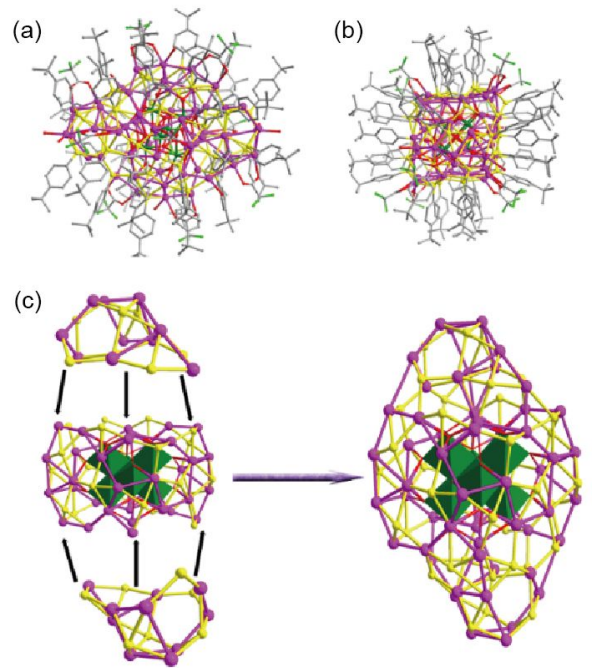

图 $11(\mathrm{a}, \mathrm{b})$ 银硫簇合物 $\left[\mathrm{Mo}_{6} \mathrm{O}_{22} @ \mathrm{Ag}_{58} \mathrm{~S}_{2}\left(\mathrm{SC}_{6} \mathrm{H}_{4} \mathrm{Bu}^{t}\right)_{36}\left(\mathrm{CF}_{3}-\right.\right.$ $\mathrm{COO})_{10}\left(\mathrm{H}_{2} \mathrm{O}\right)_{8}$ ] 从不同方向看的球棍分子结构; (c) 2 个 $\mathrm{Ag}_{10} \mathrm{~S}_{6}$ 加盖在腰部 $\mathrm{Ag}_{38} \mathrm{~S}_{26}$ 上下两端形成的芒果型的 $\mathrm{Ag}-\mathrm{S}$ 外壳结构 (网络版彩图) 


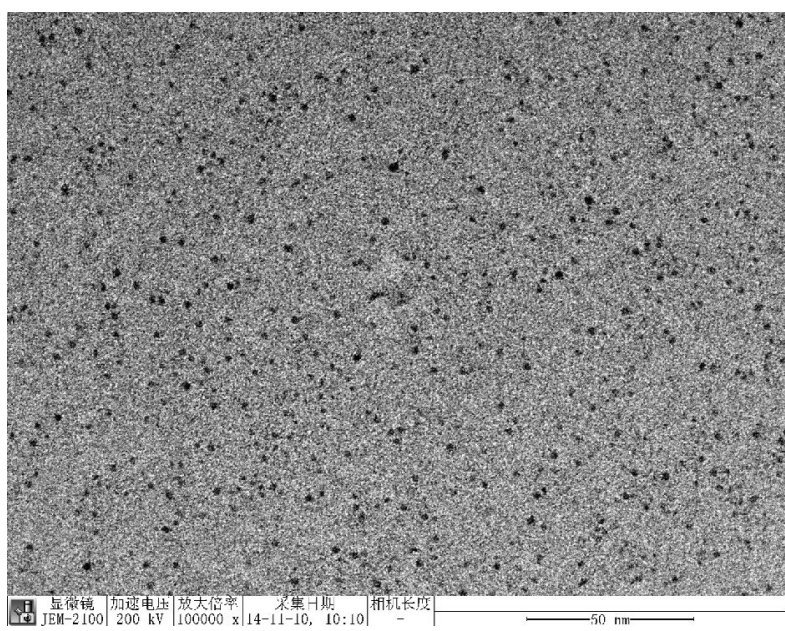

图 $12\left[\mathrm{Mo}_{6} \mathrm{O}_{22} @ \mathrm{Ag}_{58} \mathrm{~S}_{2}\left(\mathrm{SC}_{6} \mathrm{H}_{4}{ }^{t} \mathrm{Bu}\right)_{36}\left(\mathrm{CF}_{3} \mathrm{COO}\right)_{10}\left(\mathrm{H}_{2} \mathrm{O}\right)_{8}\right]$ 的高 分辨率的透射电镜图

由于容易形成软的金属氧酸盐, 因此形貌分析容易看 到超结构, 然而目前仅观察到分立结构而非超结构, 这暗示当外围包裹着硫醇外壳时, 内部包裹着含氧多 酸的内壳没有可能长成一个软的含氧多酸的超结构.

\section{3 银硫纳米簇合物的发光性能}

目前, 已合成的银硫纳米簇合物多为零维结构, 分 子尺寸大小都是纳米级, 由于量子限域效应而产生独 特的物理性质, 以至于在荧光功能材料方面的研究引 起了化学工作者的广泛关注.

2010年, 王泉明等 ${ }^{[6]}$ 测试了所合成的银硫纳米簇 合物 $\left[\mathrm{Ag}_{62} \mathrm{~S}_{13}\left(\mathrm{SBu}^{t}\right)_{32}\right]\left(\mathrm{BF}_{4}\right)_{4}$ (简称 $\mathrm{Ag}_{62}$ ) 的光学性质. 该 $\mathrm{Ag}_{62}$ 纳米簇合物的电子吸收光谱在紫外区显示两个 肩峰, 在可见区域有一个吸收峰(图13(a)). 可见光的 $543 \mathrm{~nm}$ 低能带的吸收主要归属于 $\mathrm{S} 3 \mathrm{p} \rightarrow \mathrm{Ag} 5 \mathrm{~s}$ 的电子 转移, 这与假想的 $\mathrm{Ag}_{2} \mathrm{~S}$ 的跃迁是相似的. 在甲醇溶液 中, 该族合物的激发光谱在紫外区域显示了一个高能 的吸收带和两个稍微低的吸收带(497和591 nm). 吸收 和激发光谱的不同点说明跃迁三重态是禁阻的. 激发 态的三重态的本质由发光寿命决定. 在室温下, 该固 态簇合物无论在紫外光或可见光激发, 在 $621 \mathrm{~nm}$ 处发 射出亮红光(荧光寿命 $31.7 \pm 9 \mu \mathrm{s}$ ), 而在溶液中荧光发 射峰位移至 $613 \mathrm{~nm}$ (荧光寿命 $1.5 \pm 0.2 \mu \mathrm{s}$ ) 处. 该簇合物 发射的红光暂且归属于受到 $\mathrm{Ag} \cdots \mathrm{Ag}$ 连接影响的从 $\mathrm{S}$ $3 \mathrm{p} \rightarrow \mathrm{Ag} 5 \mathrm{~s}$ 的跃迁. 该纳米簇合物的甲醇溶液可以在 室温下保存数个月后仍然发出较强的红色苂光, 如图

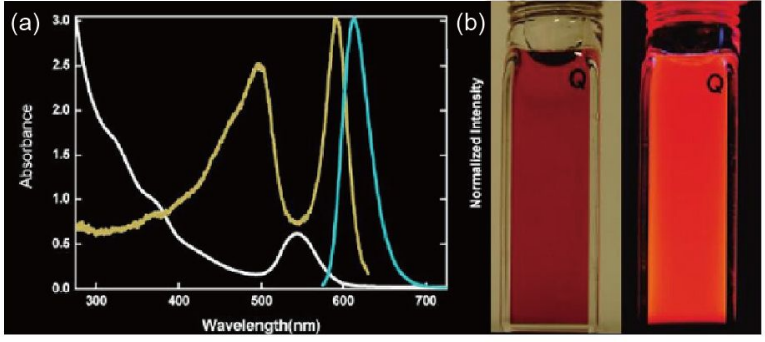

图 13 (a) $\mathrm{Ag}_{62}$ 纳米簇合物的电子光谱 (白色线), 激发光谱 (黄色线)和发射光谱(蓝色线); (b) 室温下分别在自然光条件 下(左侧)和在 $365 \mathrm{~nm}$ 激发波长下(右侧)甲醇溶液中 $\mathrm{Ag}_{62}$ 纳米 簇合物的发光照片(网络版彩图)

13(b) 所示.

值得一提的是, 安徽大学的朱满洲等 ${ }^{[28,29]}$ 最近研究 了一个结构上与 $\left[\mathrm{Ag}_{62} \mathrm{~S}_{13}\left(\mathrm{SBu}^{t}\right)_{32}\right]^{4+}$ 非常相似的阳离子 簇 $\left[\mathrm{Ag}_{62} \mathrm{~S}_{12}\left(\mathrm{SBu}^{t}\right)_{32}\right]^{2+}$. 二者区别在于 $\left[\mathrm{Ag}_{62} \mathrm{~S}_{12}\left(\mathrm{SBu}^{t}\right)_{32}\right]^{2+}$ 的簇中心少了一个硫原子, 然而其对应的荧光性能却 发生了显著的猝灭. 通过飞秒瞬态吸收光谱和含时密 度泛函计算将所观察到的荧光猝灭归属于硫原子的 缺失导致结构出现了自由价电子, 进而改变了配体到 金属的电荷转移(LMCT, S 3p $\rightarrow \mathrm{Ag} 5 \mathrm{~s}$ ).

2013年, 苏忠民等 ${ }^{[15]}$ 研究了其合成的 20 核银簇 $\left[\mathrm{Ag}_{20}\left(\mathrm{SBu}^{t}\right)_{10}\left(\mathrm{CF}_{3} \mathrm{COO}\right)_{2}\right] \mathrm{Cl} \cdot\left(\mathrm{CF}_{3} \mathrm{COO}\right)_{7} \cdot 5 \mathrm{CH}_{3} \mathrm{OH}$ (简称 $\left.\mathrm{Ag}_{20} \mathrm{~S}_{10}\right)$ 的发光性质: 该 $\mathrm{Ag}_{20} \mathrm{~S}_{10}$ 簇合物的甲醇溶液在 $330 \mathrm{~nm}$ 激发下, 于 $410 \mathrm{~nm}$ 处显示出蓝光发射带. 进一 步比较叔丁基硫银配体在甲醇溶液的发射, 推断出 $\mathrm{Ag}_{20} \mathrm{~S}_{10}$ 纳米簇的发射起源于 $\mathrm{S} 3 \mathrm{p}$ 到 $\mathrm{Ag}$ 5s 的电荷转移, 扰乱了 $\mathrm{Ag} \cdots \mathrm{Ag}$ 作用, 有效增强了簇合物的苂光发射 强度。

2013年, 孙頔等 ${ }^{[17]}$ 通过大气中 $\mathrm{CO}_{2}$ 的固定, 以碳酸 根离子为模板诱导形成了一个温度敏感的荧光纳米簇 $\left[\left(\mathrm{CO}_{3}{ }^{2-}\right) @ \mathrm{Ag}_{20}\left(\mathrm{SBu}^{t}\right)_{10}(\mathrm{DMF})_{6}\left(\mathrm{NO}_{3}\right)_{8}\right]$ (简称 $\left.\mathrm{Ag}_{20}\right)$. 这个 $\mathrm{Ag}_{20}$ 纳米簇合物在室温下没有显示出明显的发射峰, 但是在 $77 \mathrm{~K}$ 下, $\mathrm{Ag}_{20}$ 纳米簇发绿光, 低温不仅增强了苂 光的发射强度, 而且苂光发射峰还发生了红移(图 14). 由于它的可目测的温度敏感的发射光强度的变化, 可 以考虑作为潜在的温度计传感器. 在 $77 \mathrm{~K}$, 该银族合 物发射的绿光是以簇为中心的跃迁结合配体到金属 $(\mathrm{S} 3 \mathrm{p} \rightarrow \mathrm{Ag} 5 \mathrm{~s})$ 的电荷转移. 在簇合物中出现的 $\mathrm{Ag} \cdots \mathrm{Ag}$ 距离的冷收缩导致了随着温度的降低, 刚性的增强和 快速地隙间窝越, 这将有效地减少由于非辐射跃迁引 

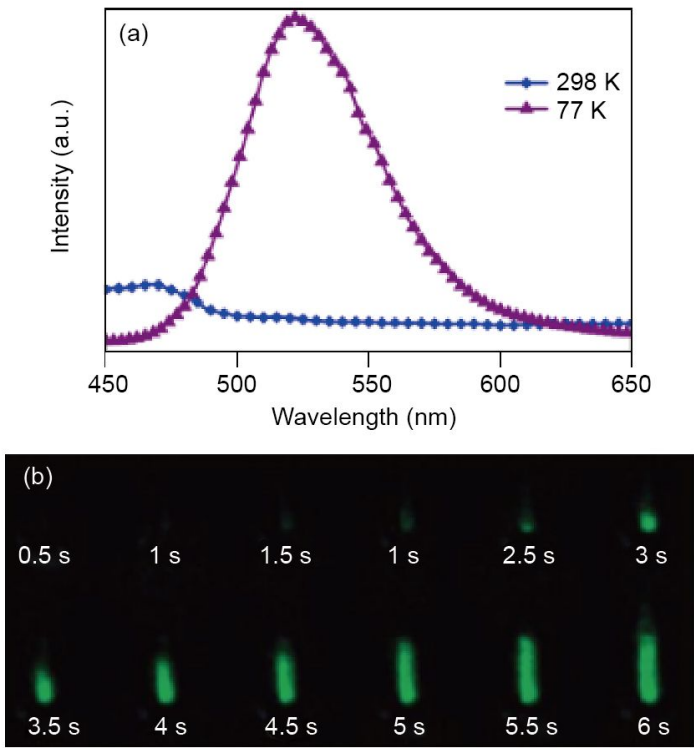

图 14 (a) 在 $365 \mathrm{~nm}$ 波长激发下, $\mathrm{Ag}_{20}$ 纳米簇合物在 77 和 $298 \mathrm{~K}$ 时的发射光谱; (b) 将 $\mathrm{Ag}_{20}$ 纳米簇合物放入液氮 $6 \mathrm{~s}$ 后用 手提紫外灯 (365 nm) 照射后从下到上逐渐的光激发照片(网 络版彩图)

起的能量转移. 因此, 以簇为中心的发射随着温度从 298 到 $77 \mathrm{~K}$ 的降低而增强.

2013 年, 孙頔等 ${ }^{[18]}$ 所合成的另外一个以 $S^{2-}$ 为阴 离子模板诱导形成的球形银硫簇合物 $\left[\left(\mathrm{S}^{2-}\right) @ \mathrm{Ag}_{56} \mathrm{~S}_{12-}\right.$ $\left.\left(\mathrm{SBu}^{t}\right)_{20}\right]\left(10 \mathrm{OAc} \cdot 6 \mathrm{DMF} \cdot \mathrm{CH}_{3} \mathrm{OH}\right)$ (简称 $\mathrm{Ag}_{56}$ ) 在固态及室 温下, 没有明显的发射峰出现, 但在 $77 \mathrm{~K}$ 下发射出亮红 色的光(图15). 在 $77 \mathrm{~K}$ 及在一系列的激发波长(300 500 $\mathrm{nm})$ 下, 该簇合物在 $623 \mathrm{~nm}$ 处显示出最大的发射峰. 这 个发射可以归属于 $\mathrm{Ag} \cdots \mathrm{Ag}$ 连接干扰的配体到金属 $(\mathrm{S}$ $3 \mathrm{p} \rightarrow \mathrm{Ag} 5 \mathrm{~s}$ ) 的电荷转移. 温度的降低, 刚性的增强和快 速地隙间穿越, 这将有效地减少由于非辐射跃迁引起 的能量转移.

2014年, 藏双全等 ${ }^{[19]}$ 测试了所报道硝酸根离子为 模板诱导的鸟巢形的银硫纳米簇合物 $\left[\mathrm{Ag}_{33} \mathrm{~S}_{3}\left(\mathrm{SBu}^{t}\right)_{16^{-}}\right.$ $\left.\left(\mathrm{CF}_{3} \mathrm{CO}_{2}\right)_{9}\left(\mathrm{NO}_{3}\right)\left(\mathrm{CH}_{3} \mathrm{CN}\right)_{2}\right]\left(\mathrm{NO}_{3}\right)$ (简称 $\left.\mathrm{Ag}_{33}\right)$ 的固体荧光 发射图, 发现该 $\mathrm{Ag}_{33}$ 纳米簇展示了一个荧光峰的位置 随温度变化的性质(图16(a,b)). 当温度从298 77 K变 化时, 荧光颜色从黄色变为橙色, 从低温到高温时, 橙 色发光变为黄色, 表明此化合物的热致荧光是可逆的, 图16(d)是最大发射峰的波长和强度分别与温度的关系 图. 此现象与硝酸根模板的配位模式、 $\mathrm{Ag} \cdots \mathrm{S}$ 和 $\mathrm{Ag} \cdots$ $\operatorname{Ag}$ 随温度的变化情况有关.

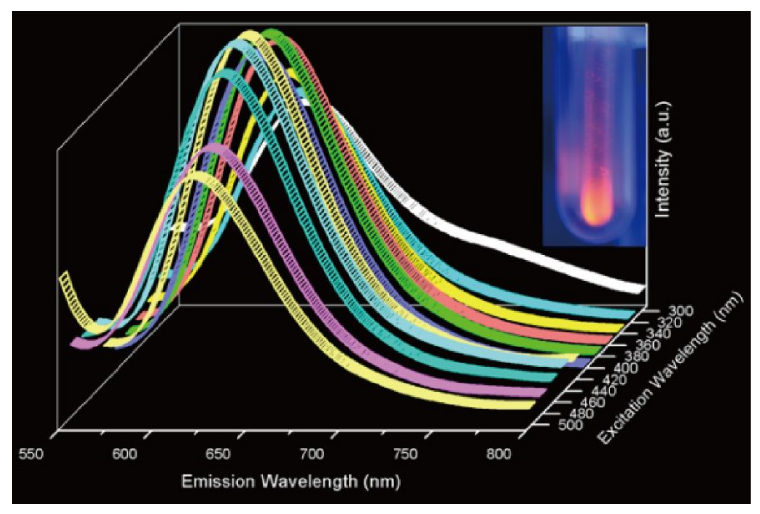

图 15 在 $77 \mathrm{~K}$, 不同激发波长下, $\mathrm{Ag}_{56}$ 纳米簇的发射光谱图. 插图: 将 $\mathrm{Ag}_{56}$ 簇放入液氮中后用手提紫外灯 $(365 \mathrm{~nm}$ 激发波 长)照射下 $\mathrm{Ag}_{56}$ 簇的光激发发光图片(网络版彩图)
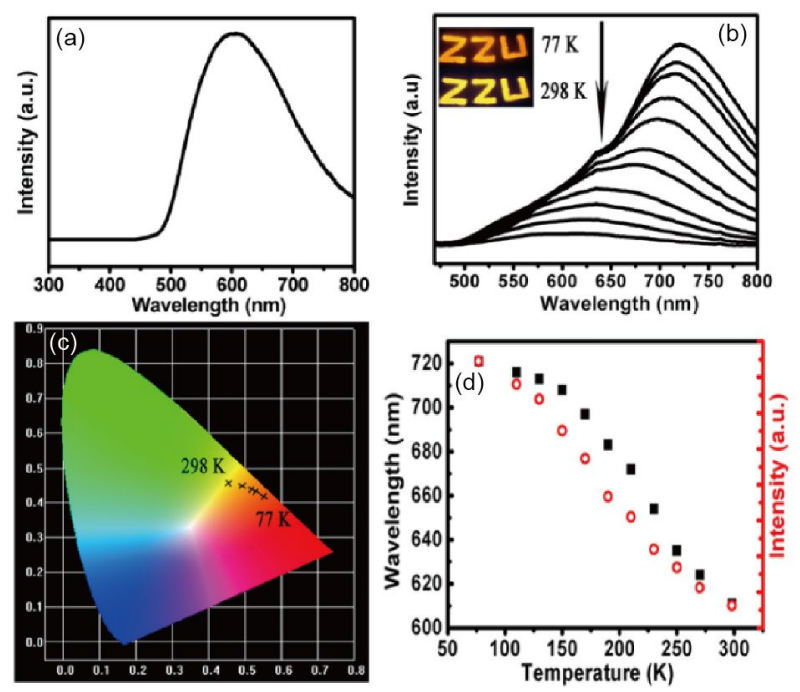

图 16 (a) $\mathrm{Ag}_{33}$ 纳米簇合物的室温固体苂光图(368 nm激发 波长); (b) 从77 298 K 的变温荧光图; (c) 变温下对应的国际 照明委员会 (CIE) 色度图; (d) 最大发射峰的波长和强度分别 与温度的关系图(网络版彩图)

\section{3 结论}

目前, 具有新颖结构和性能的银硫纳米簇合物的 组装合成引起了人们浓厚的研究热情. 这类簇合物的 形成, 往往涉及多个组分的组装过程, 其合成富有挑 战性. 在本课题组近几年的工作中, 发现阴离子模板 剂在诱导硫醇银簇合物的自组装过程中显示出了巨 大的优势. 在简单无机阴离子以及复杂多酸阴离子的 辅助下, 合成了一系列结构新颖的银硫纳米簇合物. 这类簇合物的结构大多为内嵌各类阴离子的笼状构 
型, 内嵌离子的形状和大小影响形成的银硫族合物的 结构.

此外, 自从王泉明等 ${ }^{[6]}$ 研究了具有苂光性能的银 硫纳米簇合物以来, 现在对于银硫纳米簇发光性能的 研究还处于初始阶段, 室温下具有苂光性能的银硫簇 很少报道, 一些银硫簇合物仅在 $77 \mathrm{~K}$ 表现出苂光发射
性能. 所报道的银硫簇合物的发光属性大多都是配体 到金属的电荷转移和以金属簇为中心的电荷转移. 阴 离子为模板内嵌在簇合物中心对发光性质有无显著 的影响还有待进一步研究. 相信未来经过化学工作者 的努力, 银硫纳米簇合物的合成规律、结构及发光性 能间的构-效关系将会逐渐被揭开和掌握.

\section{参考文献}

1 Wang QM, Lin YM, Liu KG. Acc Chem Res, 2015, 48: 1570-1579

2 Liu H, Song CY, Huang RW, Zhang Y, Xu H, Li MJ, Zang SQ, Gao GG. Angew Chem Int Ed, 2016, 55: 3699-3703

3 Fuhr O, Dehnen S, Fenske D. Chem Soc Rev, 2013, 42: 1871-1906

4 Xie YP, Jin JL, Duan GX, Lu X, Mak TCW. Coordin Chem Rev, 2017, 331: 54-72

5 Corrigan JF, Fuhr O, Fenske D. Adv Mater, 2009, 21: 1867-1871

6 Li G, Lei Z, Wang QM. J Am Chem Soc, 2010, 132: 17678-17679

7 Li XY, Su HF, Yu K, Tan YZ, Wang XP, Zhao YQ, Sun D, Zheng LS. Nanoscale, 2015, 7: 8284-8288

Müller A, Kögerler P, Dress AWM. Coordin Chem Rev, 2001, 222: 193-218

Busch DH. J Incl Phenom Macrocycl Chem, 1992, 12: 389-395

0 Diederich F, Stang PJ. Templated Organic Synthesis. Weinheim: Wiley-VCH, 2000

1 Rais D, Yau J, Mingos DMP, Vilar R, White AJP, Williams DJ. Angew Chem Int Ed, 2001, 40: 3464-3467

2 Rais D, Mingos DMP, Vilar R, White AJP, Williams DJ. J Organom Chem, 2002, 652: 87-93

3 Liu CW, Chang HW, Fang CS, Sarkar B, Wang JC. Chem Commun, 2010, 46: 4571-4573

4 Liu X, Yang H, Zheng N, Zheng L. Eur J Inorg Chem, 2010, 2010: 2084-2087

Zhou K, Wang XL, Qin C, Wang HN, Yang GS, Jiao YQ, Huang P, Shao KZ, Su ZM. Dalton Trans, 2013, 42: 1352-1355

Zhou K, Qin C, Wang XL, Shao KZ, Yan LK, Su ZM. CrystEngComm, 2014, 16: 7860-7864

Yuan S, Deng YK, Wang XP, Sun D. New J Chem, 2013, 37: 2973-2977

8 Sun D, Wang H, Lu HF, Feng SY, Zhang ZW, Sun GX, Sun DF. Dalton Trans, 2013, 42: 6281-6284

9 Li B, Huang RW, Qin JH, Zang SQ, Gao GG, Hou HW, Mak TCW. Chem Eur J, 2014, 20: 12416-12420

2 Zhou K, Qin C, Li HB, Yan LK, Wang XL, Shan GG, Su ZM, Xu C, Wang XL. Chem Commun, 2012, 48: 5844-5846

1 Huang RW, Xu QQ, Lu HL, Guo XK, Zang SQ, Gao GG, Tang MS, Mak TCW. Nanoscale, 2015, 7: 7151-7154

2 Li XY, Tan YZ, Yu K, Wang XP, Zhao YQ, Sun D, Zheng LS. Chem Asian J, 2015, 10: 1295-1298

Balraj V, Vidyasagar K. Inorg Chem, 1999, 38: 1394-1400

Wang JP, Du XD, Niu JY. J Solid State Chem, 2006, 179: 3260-3264

Dai L, Wang E, You W, Zhang Z. J Clust Sci, 2008, 19: 511-519

Pope MT. Heteropoly and Isopoly Oxometalates. New York: Springer, 1983

Su ZH, Zhou BB, Zhao ZF, Zhang X. Inorg Chem Commun, 2008, 11: 334-337

8 Jin S, Wang S, Song Y, Zhou M, Zhong J, Zhang J, Xia A, Pei Y, Chen M, Li P, Zhu M. J Am Chem Soc, 2014, 136: 15559-15565

29 Jin S, Wang S, Xiong L, Zhou M, Chen S, Du W, Xia A, Pei Y, Zhu M. Chem Mater, 2016, 28: 7905-7911 


\title{
Synthesis, structures and luminescence of silver (I) thiolate nanoclusters based on anion templates
}

\author{
Genggeng Luo ${ }^{1}$, Zhi Wang ${ }^{2}$, Li-Ping Cheng ${ }^{1,2}$, Quan-Qin Zhao ${ }^{2}$, Xing-Po Wang ${ }^{2}$, Di Sun ${ }^{2 *}$ \\ ${ }^{1}$ College of Materials Science and Engineering, Huaqiao University, Xiamen 361021, China \\ ${ }^{2}$ School of Chemistry and Chemical Engineering, Shandong University, Jinan 250100, China \\ *Corresponding author (email: dsun@sdu.edu.cn)
}

\begin{abstract}
The construction of silver (I) thiolate nanoclusters is challenging because they involve the assembly of multiple components. Anionic template method is a simple and effective method for the synthesis of silver (I) thiolate nanoclusters, especially for the control of their structures. In this mini review, we briefly summarizes the recent progress in the synthesis, crystal structures and luminescence of silver (I) thiolate nanoclusters, which template by simple ball-like anions (halogen and $\mathrm{S}^{2-}$ ), trigonal anions $\left(\mathrm{CO}_{3}{ }^{2-}\right.$ and $\left.\mathrm{NO}_{3}{ }^{-}\right)$, even complex POM anions $\left(\left[\mathrm{W}_{6} \mathrm{O}_{21}\right]^{6-}\right.$ and $\left.\mathrm{Mo}_{6} \mathrm{O}_{22}{ }^{8-}\right)$. The size and type of anion templates play important roles in shaping the final silver (I) thiolate nanoclusters.
\end{abstract}

Keywords: silver thiolate nanoclusters, crystal structures, anion templates, luminescence

doi: $10.1360 / \mathrm{N} 032016-00238$ 